\title{
Brecha de instalación de políticas públicas: chile crece contigo y trabajo social
}

\author{
Adriana Sanhueza Cisterna*
}

\begin{abstract}
El presente artículo es el resultado de una investigación documental sobre la implementación del Programa Chile Crece Contigo. La investigación se realizó sobre el recorrido que efectúa la política pública que va desde el Gobierno Central a las comunas, reflexionando sobre su proceso de implementación. La intención es aportar a la valoración de la política no por su apariencia primera, sino por su implementación, otorgando elementos de análisis de proceso, que den algunas garantías conceptuales, reflexivas y una panorámica de su impacto. Finalmente, se establece que aun cuando este programa tiene una base teórica importante, hay temas pendientes debido a la disponibilidad de capacidades técnicas y profesionales para su implementación a nivel municipal.
\end{abstract}

Palabras clave: Descentralización / Municipalismo / Género / Trabajo Social.

\section{Brecha de instalação de políticas públicas: chile cresce contigo e trabalho social}

RESUMO

O presente artigo é o resultado de uma investigação documental sobre a implementación do Programa Chile Cresce Contigo. A investigação realizouse sobre o percurso que efectua a política pública que vai desde Governo Central às comunas, reflexionando sobre seu processo de implementación. A intenção é contribuir à valoração da política não por sua aparência primeira, senão por sua implementación, outorgando elementos de análises de processo, que dêem algumas garantias conceptuais, reflexivas e uma panorámica de seu impacto. Finalmente, estabelece-se que ainda que este programa tem uma base teórica importante há temas pendentes devido à disponibilidad de capacidades técnicas e profissionais para sua a implementación a nível municipal.

Palavras chave: Descentralización / Municipalismo / Género / Trabalho Social.

* Chilena, trabajadora social. Magíster en Gobierno y Gerencia Pública. Docente Escuela de Trabajo Social, Universidad Católica Silva Henríquez.

Correo electrónico: asanhueza@ucsh.cl 


\section{The distance of installation of public politics: chile grows with you and social work}

\section{ABSTRACT}

The present article is the result of a documentary investigation on the implementation of the Program Chile Grows With you. The investigation realized on the route that effects the public politics that goes from Central Government to the comunas, reflexionando on his process of implementation. The intention is aportar to the assessment of the politics no by his apariencia first, but by his implementation, awarding elements of analysis of process, that give some conceptual guarantees, reflexivas and a panoramic of his impact. Finally, estabelece- than ainda that this program tem uma important theoretical base há subjects pendentes devido à availability of technical capacities and profissionais for sua to implementation to nível municipal.

Key words: Decentralization, Municipalism, Gender, Social Work. 


\section{Introducción}

Las políticas públicas implementadas en el último tiempo, han tenido dos focos de atención, por una parte medición de impacto y por otra el gasto que generan y los reportes que producen, sin embargo, aun cuando estas no han sido favorables para su continuidad, han proseguido sin mayores cambios de estructura.

La implementación de estas Políticas Públicas en los últimos años de Gobierno, es el momento menos referido y analizado para conocer su impacto final. Por otra parte, en el despliegue de la mayoría de estas políticas se ocupa la fuerza laboral de profesionales de las Ciencias Sociales y en particular la de Trabajadores Sociales.

El trabajo que a continuación se desarrolla es una investigación en la cual se intenta explicar a través de una política pública "Chile Crece Contigo", la importancia del Trabajo Social para la implementación exitosa de ésta, y de qué manera la creación de un diseño centralizado de una política puede determinar las brechas en su implementación.

\section{Antecedentes}

El programa Chile Crece Contigo, nace por una petición expresa de la Presidenta de la República Michelle Bachelet, para reformar las políticas de Infancia en Chile. El objetivo de esta política pública, es generar un sistema de protección social en materia de primera infancia en Chile.

La propuesta de trabajo se realiza a través de una comisión, cuyo producto final, "El Futuro de los Niños es Siempre Hoy", se ocupa como base para la generación de dicha política pública. Esta política, incluye a niños y niñas hasta los 10 años de edad. Las propuestas elaboradas en el informe fueron recogidas y analizadas por el Consejo de Ministros de Infancia, el cual entregó a la Presidenta Bachelet un informe en el que propusieron un conjunto de acciones de Gobierno para la implementación de un Sistema de Protección Integral a la Infancia, materializadas en el Chile Crece Contigo.

El sistema se enmarca dentro de la "Política Nacional y el Plan de Acción Integrado a favor de la Infancia y la Adolescencia 2001-2010". 
El propósito del programa Chile Crece Contigo, es acompañar, proteger y apoyar integralmente a todos los niños y niñas y a sus familias, a través de la prestación de servicios de carácter universal, focalizando apoyos especiales a aquellos casos que presentan una mayor vulnerabilidad: "a cada quien según sus necesidades".

El programa Chile Crece Contigo toma como referencia el consenso científico que existe sobre los buenos resultados de la intervención en la primera etapa de desarrollo de los infantes, donde se modelan las características físicas, cognitivas y psicológicas que se consolidarán en sucesivas etapas de desarrollo del niño o la niña. La neurociencia y otras ciencias asociadas demuestran que en la primera infancia existen oportunidades de desarrollo que no se repiten en otras etapas de la vida, es así como el programa Chile Crece Contigo apunta a intervenir en este nivel de desarrollo.

El modelo de gestión del programa Chile Crece Contigo se basa en un sistema de eficiencia y eficacia, de todos los recursos independiente de su fuente o niveles sectoriales en que se localicen. Supone entonces, un sistema de coordinación en red de todos los recursos (institucionales, humanos, financieros, públicos, privados, comunitarios y de las familias), disponibles para el sistema en todos los ámbitos sectoriales y territoriales que lo componen.

El enfoque del modelo de gestión propuesto por el Consejo Asesor Presidencial es orientado a resultados finales de la intervención, supone la existencia de metas globales de desarrollo de los niños y niñas, a través del ciclo de vida, estándares de calidad para todas las prestaciones del sistema, mecanismos de acreditación y fiscalización de los mismos, instrumentos de evaluación de efectividad de las prestaciones y de la política más globalmente e instrumentos de rendición de cuentas de todos los participantes.

El modelo institucional propuesto por el Consejo Asesor Presidencial, realiza un consorcio con una serie de instituciones públicas que se relacionan con la política pública como ministerios sectoriales, e instituciones de nivel central, regional, provincial y comunal, con funciones e instrumentos no coordinados, y una serie de prestadores públicos y privados que den servicios de calidad no acreditada a niños, niñas y sus familias. En la búsqueda de un organismo de alto nivel, los especialistas del Consejo Asesor responsabilizan de la ejecución del programa Chile Crece Contigo al Ministerio de Planificación Nacional (MIDEPLAN), debido a 
su carácter no sectorial, y la gran experiencia de esta institución en gestión de redes sociales, destacando tres funciones: 1) Formulación, fijación de la política pública y formulación de planes y programas, definición de criterios de implementación, acceso y evaluación. 2) Provisión de prestaciones orientadas a la infancia para responder a los objetivos de la política. 3) Fiscalización del cumplimiento de las obligaciones establecidas en leyes, reglamentos y normas complementarias a éstas.

El Consejo además propuso que cada uno de los organismos relacionados con la aplicación del programa Chile Crece Contigo, se rija por las normas de control de gestión de la Dirección de Presupuesto (DIPRES). Fortaleciendo su Sistema de Información para la Gestión Institucional (SIG), que forma parte de los Programas de Mejoramiento de la Gestión (PMG).

La primera de estas líneas es la provisión de nuevas prestaciones de apoyo a la gestión en Red Local en convenio con Municipalidades acreditadas, transfiriendo recursos desde las Secretarías Regionales Ministeriales, a través de convenios que establezcan responsabilidades, condiciones y contenidos de las prestaciones y servicios, contra programas de trabajo anuales. Las redes municipales están formadas por los centros de salud, centros preescolares y escuelas, y otros prestadores locales, vinculadas con el Municipio a través de Oficinas Locales de Infancia, rindiendo los recursos traspasados anualmente, a MIDEPLAN, este mismo Ministerio es a su vez quien realiza la identificación de los beneficiarios de prestaciones.

\section{Conclusiones del estudio}

\section{De la temática general}

- Debate sobre el tema de las políticas públicas de nivel central en relación a la descentralización y funcionamiento municipal.

Es posible mencionar que el modo de formulación de políticas públicas existente en Chile, y la forma de cómo estas se equilibran con la descentralización del país, sacan a la luz las fallas del modelo de descentralización, ya que se torna solo un discurso especialmente en el nivel municipal, pues las políticas públicas se definen centralmente, no teniendo en muchos casos un correlato con lo territorial. Al mencionar la heterogeneidad de los distintos 
territorios en materia de pobreza, sea esta urbana o rural, o según los distintos tipos de gestión municipal, tenemos un cuadro muy diverso para la aplicación de un formato único de política pública, por lo tanto, cuando nos referimos a descentralización estamos hablando de municipios que deben aplicar con sus particulares condiciones una política pública rígida ya establecida (Palma y Rufián, 1989).

El diseño de políticas públicas se ve enfrentado a una condición de heterogeneidad en la aplicación y centralidad extrema en el diseño.

Por otra parte, la posibilidad de una buena o coherente implementación de estas políticas públicas depende de la capacidad de los entes que las implementan, en este caso los municipios. En algunos casos, estos funcionan de manera eficiente, porque pueden complementar con sus propios recursos y capacidades de personal existente o de contratación de nuevos trabajadores los mandatos del nivel central.

En lo que respecta al factor económico y cómo se descentralizan los recursos para estas políticas, es posible mencionar, a la luz de su implementación, que en general todas las transferencias económicas realizadas por los ministerios involucrados son determinadas por el nivel central, tomando como base condicionantes programáticas de este sistema, lo que no permite movilidad de carácter programático.

A pesar de ser el eslabón principal en la aplicación de políticas y el terreno de la implementación, el nivel municipal es la parte inferior de la pirámide en la toma de decisiones, pues es privilegio de determinados sectores e intereses, que instala en el aparato estatal nuevas tensiones, en tanto privilegia automáticamente a los niveles centrales u organismos oficiales, lugar donde se encuentra la toma de decisiones, en detrimento de otros, que están de cara a los usuarios o beneficiarios, como es el caso de las municipalidades.

Estas políticas públicas originan dentro de la burocracia un gran número de reacomodos y ajustes, con la intención de materializar los planes. Su desarrollo tiende entonces a ajustarse más a la racionalidad dictada por los intereses que defiende la institucionalidad municipal, que a la lógica de la decisión política de los nuevos programas; desde esta perspectiva, lo que las instituciones estatales hacen no puede ser visto simplemente 
como la traducción en acción o implementación de un conjunto de normas.

Su actividad refleja más bien intentos por compatibilizar los intereses propios con las propias clientelas municipales, pues en última instancia son estas ofertas las que también asegurarán la reelección de las autoridades políticas instaladas en el poder (González, 2008).

\section{- Debatesobredescentralización administrativa yfinanciera y la concordancia con las capacidades municipales para gestionarlas.}

Con la reforma del Estado y la implementación de un modelo de nueva gestión pública, en materia municipal, las políticas públicas dependen de la voluntad y la calidad técnica que demuestren los municipios para tal implementación.

Por una parte, se pueden encontrar algunas gestiones municipales enredadas y justificadas en sus escasos recursos propios, y por otra, tomando en cuenta el amplio espectro de los 345 municipios que Chile posee, es posible encontrar municipios que tienen reales gestiones locales, traspasando el antiguo debate de administraciones municipales a gobiernos locales, con innovaciones y características propias (González, 2008).

Es el hecho de la baja y engorrosa asignación de montos concedidos a los municipios, tanto en fondos traspasados desde los Ministerios, como el dificultoso cálculo del fondo común municipal, asignaciones provenientes del Fondo Nacional de Desarrollo Regional (FNDR) y otros fondos especiales que llegan a las comunas, en que se ampara el debate del real alcance de estas administraciones municipales.

Respecto de la descentralización administrativa, entendida como la entrega de posibilidades de decisión en el ámbito municipal, sobre sus propios quehaceres y los que les competen (Nogueira y Cumplido, 1993), se puede mencionar que en materia de administración de política pública y en especial para este estudio sobre implementación del Chile Crece Contigo, ésta sólo alcanza a tocar sobre estándares de aplicación y evaluación, ya establecidos centralmente.

Por otra parte, la descentralización financiera pensada como la transferencia de recursos no condicionada, para el Chile Crece 
Contigo, no ha sido materializada en el caso particular de los 48 municipios del Gran Santiago que cuentan con el mencionado programa

Es posible mencionar que no necesariamente las cosas se solucionan con más recursos. Esto marca la discusión de todos los temas del ámbito municipal. Más recursos y también mejor gestión resuelven los problemas de funcionamiento referidos a liderazgo, administración, capacidad de recursos humanos, entre otros. No existe reforma del Estado que no tenga como debate central el tema de la cualificación de los recursos humanos (Cunill, 2005).

\section{Reflexiones sobre la temática específica del estudio}

\section{- La brecha de instalación de las políticas públicas en materia de protección social.}

La brecha en este sistema, se origina por los diversos entes que administran cada una de las etapas del programa en los diferentes niveles de la organización. Para el caso de los municipios, las características propias de estos ya otorgan brecha. A modo de ejemplo: hay municipios que han nominado una secretaría ejecutiva de protección social. Existen otros que ni siquiera nominan por decreto al encargado/a de protección social, sea esto por énfasis de la gestión o por el personal disponible, como es el caso de pequeños municipios en que un solo funcionario desarrolla todas las funciones sociales. De modo que la brecha, en un comienzo, se presenta por las enormes diferencias que existen entre los propios municipios.

\section{- Sobre el marco referencial del Chile Crece Contigo y la temática de género}

Según los entrevistados, el Chile Crece Contigo, contribuye en la temática de género porque "apoya a la mujer en la crianza de los niños o niñas", situación que difiere de la teoría de género, que visibiliza la temática del cuidado de los niños y niñas como una forma de excluir a la mujer del mercado laboral y de la toma de decisiones en el ámbito de lo público.

En materia de políticas de género en nuestro país, el órgano encargado de proponer, dar seguimiento y resguardar este 
afluente es el Servicio Nacional de la Mujer (SERNAM), creado en el año 1991, el cual pasa de un sostenido plan de igualdad de oportunidades a una agenda gubernamental de políticas de género ya en los últimos años de gestión gubernamental.

La relevancia que ha tenido el Chile Crece Contigo, y el esperado impacto en la vida de las personas, las familias y las mujeres, es materia de análisis, pues esta política ha generado grandes expectativas, y es el Consejo quien fundamenta la propuesta por el impacto que esto tendrá en la vida de los futuros adultos de Chile.

Sin embargo cabe preguntarse ¿Cuál es el rol de las mujeres para este esperado estado de desarrollo?

El rol de las mujeres en el Chile Crece Contigo ha sido uno de los puntos más cuestionables de esta política, puesto que la implementación del programa en todo momento pone a la madre, o a la mujer en el rol de cuidadora, al centro de la responsabilidad del maternaje y del rol de la familia que cobija y recibe a este niño o niña, vale decir, que en materia de igualdad de responsabilidades en la crianza de los hijos/as esta política retrocede o consolida la ya sabida tradición de asignar el cuidado de los pequeños a las mujeres.

Cabe mencionar, que el Consejo presentó algunas propuestas con respecto al cambio del pre y el post natal para el cuidado de los niños o niñas, pero el ejecutivo no avanzó en profundizar esta propuesta, con la idea de trasladar estos cuidados a los padres de las criaturas, cuando así la pareja lo considerase.

Entonces, a pesar del impacto esperado que tendría esta política pública en el desarrollo de los niños y niñas en el presente inmediato, esta política en materia de maternidad retrocede al rol histórico de la mujer en el cuidado de los hijos e hijas, con los consabidos impactos sociales que esto tiene, en pro del desarrollo de los niños y niñas.

\section{- Sobre cómo estas visiones de mundo, plasmadas en una política pública, se convierten en actividades cotidianas de la familia chilena.}

El impacto de cambios en los roles de género, fruto de una creciente participación política y laboral de las mujeres, hacen difícil compatibilizar la vida laboral y familiar, situaciones propias 
de los roles femeninos tradicionales realizados en el ámbito doméstico (cuidado de niños, de adultos mayores, de personas postradas o discapacitadas), con las exigencias laborales. Lo anterior, constituye la base para la creación de políticas públicas como el Chile Crece Contigo, liberando a las familias de la excesiva carga de responsabilidades, así como del cuidado y protección de los miembros más dependientes de éstas.

Chile es el país que presenta a nivel latinoamericano la tasa más baja de participación laboral femenina, aun cuando la tasa de actividad de las mujeres está creciendo velozmente como lo revela la sistemática incorporación de mujeres al mercado laboral, especialmente entre los 18 y 25 años de edad, en plena edad fértil. ${ }^{1}$

Es así que políticas públicas y más aún un sistema de protección social como el que en la actualidad tiene el país, otorga un soporte familiar más allá de lo económico, centrándose en entender la seguridad por el solo hecho de ser ciudadano o ciudadana nacido en el territorio de Chile. Es preciso también mencionar de qué manera esta situación reordena las relaciones familiares, repone el rol de la mujer como cuidadora y criadora, y a la vez, otorga al Estado un rol de co-cuidador, para que la mujer pueda aportar en lo que en materias económicas respecta, sea en lo familiar como en el aporte que realiza al crecimiento del país.

Todo ello en un contexto de transformaciones en la organización familiar y la coexistencia de una diversidad de tipos de familias para las que no existen respuestas adecuadas: conviven familias que responden al modelo tradicional biparental, con familias de jefatura femenina, convivencias, familias unipersonales especialmente de mujeres adultas mayores solas, entre las más frecuentes. Los hogares nucleares (biparentales con hijos) representan el 57\% del total de familias, mientras que el $43 \%$ restante representa otros tipos de familias (Hardy 2006).

De modo que tanto el sistema de protección social, como el Chile Crece Contigo, no realizan distinciones la hora de entregar sus prestaciones, solo se accede a servicios más específicos vía ficha de protección social, traspasando la barrera del tipo de familia desde la que el beneficiario proviene, para la entrega de algún tipo de ayuda, situación que cambia la legitimidad e ideación del

1 Inequidades de Género en el Mercado Laboral, en cuaderno de investigación №35, División de Estudios, Dirección del Trabajo, Santiago, Chile (2008). 
tipo de familia proveniente en relación a quienes buscan algún tipo de soporte.

\section{- Trabajo social y participación en políticas públicas}

En general, es posible mencionar que el sistema de protección social chileno posee en su base laboral un gran número de trabajadores sociales, ampliándose en el transcurso del tiempo, en la medida que el sistema de protección social se establece como tal e incorpora más prestaciones en su plataforma.

Los Trabajadores Sociales, realizan su labor, principalmente en las bases de esta implementación, o en el nivel municipal, sin embargo, la correlación de este trabajo con la producción de conocimientos o la incorporación de sus reflexiones en cuanto a la implementación de esta u otras políticas públicas es inexistente.

En particular sobre la política del Chile Crece Contigo, en sus bases teóricas participó un conjunto de expertos tanto en el tema técnico como de implementación; no obstante, ninguno de estos tenía como profesión de origen la de Trabajador Social, lo que habla de la marginación de la disciplina, en relación al diseño y por sobre todo a su fuerza laboral.

\section{Recomendaciones}

Esta reflexión se centra primeramente en lo que respecta al tema central: brecha de instalación, y en un segundo lugar, se formulan recomendaciones específicas sobre la política pública analizada.

Es de carácter fundamental y la principal razón, que cualquiera sea la brecha de instalación de una política pública a nivel municipal, tiene relación con la diversidad de los municipios donde se despliegan estas, debido principalmente, a la heterogeneidad municipal. Existe un tratamiento homogéneo en la implementación de políticas públicas a nivel municipal, sin embargo, las realidades son muy diferentes.

En el periodo de formulación de la política, más aún en el diseño de su modelo de gestión, no se encuentran consideradas las voces de los organismos intermedios o de quienes finalmente la ejecutan, situación que ahonda la brecha de implementación.

Para el caso de la política pública estudiada, a la fecha no existe evaluación de implementación, situación que no configura la 
intensidad del impacto. Se debe revisar de manera urgente el procedimiento con el fin de perfeccionar el sistema.

Se hace necesario, por la singularidad ya mencionada del nivel municipal, dejar un margen de acción y creación o contribución propia de cada uno de los municipios, puesto que al estar tan cerrada la ejecución y del mismo modo los fondos, las diversidades municipales, sean de carácter cultural, de énfasis de la gestión, innovación u otro componente, se ven aplastadas por la política pública.

En relación a la política pública escogida para este estudio, la del Chile Crece Contigo, cabe recordar los énfasis que realizó el Consejo de la Infancia en su constatación diagnostica, y que hoy al no estar en el sistema tienen un menor nivel de impacto. Tales temas corresponden a la importancia de que esta política posea correlato con los niños/as hasta los 18 años en materia de salud, educación y/o oportunidades de inserción social.

Otro punto que quita solidez a la intervención, también señalado por el Consejo de la Infancia, es la presencia de organismos intermedios y ONG, que poseen trabajo y trayectoria específica en esta materia, que hoy no están realizando prestaciones al sistema, lo que desaprovecha su experticia y hace endeble la intervención.

En materia de igualdad de género es necesario repensar el rol que ejerce la mujer y la maternidad, como así también los cambios en lo relativo al pre, post natal y cuidado del hijo/a menor de un año, para reparar la brecha de género que esta política hoy no supera.

\section{Recomendaciones para futuras investigaciones}

El alcance de este estudio debiese ser ampliado hacia una muestra representativa que permitiera consolidar los resultados obtenidos, pudiéndose extraer aproximaciones que al cruzar variables, contribuyan a enriquecer el estudio y lograr un mayor impacto a esta investigación.

Las temáticas abordadas en este estudio, relativas al análisis de la brecha de instalación de políticas públicas, y sus relaciones a nivel teórico con materias como reforma del Estado, nueva gestión pública, descentralización y municipalismo, debieran 
tener continuidad en estudios futuros, como asimismo se deberá hacer seguimiento de las políticas públicas, en la medida que ésta se consolida y avanza.

Del mismo modo, es posible sugerir una continuidad investigativa, donde sea posible observar la implementación de las políticas públicas y hacer mediciones de impacto que podrían dar mayores argumentos analíticos en este ámbito.

\section{Referencias Bibliográficas}

CUNILL, N. (2005, Octubre). La Intersectorialidad en el Gobierno y Gestión de la Política Social. Ponencia presentada en el X Congreso Internacional del CLAD sobre la Reforma del Estado y de la Administración Pública, Santiago, Chile.

GONZALES, R. (2008). Poderes Locales, Nación y Globalización. Santiago, Chile: Ediciones Universidad Academia de Humanismo Cristiano.

HARDY, C. (2006). Hacia un Sistema de Protección Social Fundado en Derechos. Documentos Mideplan 2006. Disponible en: http://www.google.cl/url?sa=t Esource=webEcd $=1$ Eved $=0$ CBCOFjAAEurl = http\%3A\%2F\%2Fwww.mideplan.cl\%2Findex. php\%3Foption\%3Dcom_rubberdoc\%26view\%3D doc\%26id\%3D363\%26format\%3Draw\%26Itemid \%3D91 Eei=mPYYTeOCF8OC8gat4qSTCgEusg= AFQjCNFLNiuCWzbTWZ-EbswXuAn6MB4nRg.

NOGUEIRA, H. y CUMPLido, F. (1993). Derecho Político. Cuadernos Universitarios (serie manuales $\mathrm{N}^{\circ} 1$ ), Santiago, Chile: Universidad Andrés Bello.

PALMA, E. y RUFIAN, D. (1989). Los procesos de descentralización y desconcentración de las políticas sociales en América Latina. Enfoque Institucional. Santiago, Chile: ILPES. 